OPEN ACCESS

Edited by: Yaxiong Zhang,

Sun Yat-sen University Cancer

Center (SYSUCC), China

Reviewed by:

Erik Henry Knelson,

Dana-Farber Cancer Institute,

United States

Kazushige Wakuda,

Shizuoka Cancer Center, Japan

*Correspondence:

Haiquan Chen

hqchen1@yahoo.com

Yang Zhang

fduzhangyang1987@hotmail.com

${ }^{\dagger}$ These authors have contributed equally to this work

Specialty section: This article was submitted to

Thoracic Oncology,

a section of the journal

Frontiers in Oncology

Received: 29 October 2020 Accepted: 30 March 2021

Published: 19 April 2021

Citation:

Fu F, Wen Z, Gao Z, Zhao Y, Li Y, Zhang $Y$ and Chen $H$ (2021) Primary

Tumor Resection Improves Survival for EGFR-TKI-Treated Patients With Occult M1a Lung Adenocarcinoma.

Front. Oncol. 11:622723.

doi: 10.3389/fonc.2021.622723

\section{Primary Tumor Resection Improves Survival for EGFR-TKI- Treated Patients With Occult M1a Lung Adenocarcinoma}

\author{
Fangqiu Fu ${ }^{1,2,3 t}$, Zhexu Wen ${ }^{1,2,3 t}$, Zhendong Gao ${ }^{1,2,3}$, Yue Zhao ${ }^{1,2,3}$, Yuan $\mathrm{Li}^{2,3,4}$, \\ Yang Zhang ${ }^{1,2,3^{*}}$ and Haiquan Chen ${ }^{1,2,3 *}$
}

${ }^{1}$ Department of Thoracic Surgery and State Key Laboratory of Genetic Engineering, Fudan University Shanghai Cancer Center, Shanghai, China, 2 Institute of Thoracic Oncology, Fudan University, Shanghai, China, ${ }^{3}$ Department of Oncology, Shanghai Medical College, Fudan University, Shanghai, China, ${ }^{4}$ Department of Pathology, Fudan University Shanghai Cancer Center, Shanghai, China

Background: The role of primary tumor resection in occult M1a lung adenocarcinoma remains unclear, especially for patients receiving targeted therapy. The purpose of this study is to assess the effect of primary tumor resection on overall survival (OS) in lung adenocarcinoma patients with occult pleural disseminations receiving targeted therapy.

Methods: Lung adenocarcinoma patients with intraoperatively-confirmed occult pleural dissemination (M1a), who hospitalized in the Department of Thoracic Surgery in Fudan Shanghai Cancer Center from May 2008 to December 2017 and received EGFR-TKIs therapy, were enrolled. Log-rank tests were used to compare the survival differences between groups.

Results: 34 patients receiving EGFR-TKIs were enrolled. The majority of them were never smokers (29/34, 85.3\%). Among the enrolled patients, 20 (58.8\%) patients underwent primary tumor resection, while $14(41.2 \%)$ patients not. There was no distributional difference of baselines between patients undergoing and not undergoing primary tumor resection. Further analyses demonstrated that the patients undergoing primary tumor resection had a prolonged OS compared with those not (log-rank $P=0.042$ ). The 2-year and 5-year OS for patients receiving primary tumor resection and EGFR-TKIs was 90.0\% and $60.1 \%$.

Conclusions: Primary tumor resection was associated with improved survival in patients with occult intraoperatively-confirmed M1a adenocarcinoma receiving EGFR-TKIs.

Keywords: lung adenocarcinoma, M1a, primary tumor resection, EGFR-TKIs, survival

Abbreviations: BMI, body mass index; NSCLC, non-small cell lung cancer; OS, overall survival. 


\section{INTRODUCTION}

Lung cancer is the deadliest malignancy worldwide, accounting for the largest number of new cancer cases and cancer-related deaths (1). Stage IV lung cancer is responsible for $45 \%$ of newly diagnosed lung cancer patients in Surveillance, Epidemiology, and End Results (SEER) Program population-based registries (2). However, the 2-year and 5-year overall survival (OS) rate for stage IV non-small cell lung cancer (NSCLC) is only $17 \%$ and $6 \%$, respectively (3). Over the past ten years, treatment for stage IV NSCLC has been revolutionized by the rapid development of targeted therapy. In the era of targeted therapy, lung cancer patients live a much longer life than before. Nowadays lung cancer seems to be a "chronic" disease thanks to the clinical application of targeted agents. However, patients will eventually develop drug resistance to targeted agents, and it is a critical issue to address methods improving survival of patients receiving target therapy. A previous study indicated that patients with initial disease progression in primary tumors accounted for $45 \%$ of progressed patients with targeted therapy (4). Moreover, in clinical practice, occult pleural dissemination is sometimes discovered intraoperatively. There is no consensus on whether we should perform primary tumor resection in that case. Therefore, we hypothesized that surgical resection of primary tumors could improve the survival of lung adenocarcinoma patients with occult pleural metastases in the era of targeted therapy.

Although surgery is not deemed as a treatment option, given the fact that therapeutic goals of stage IV disease have focused on optimization of quality of life and palliation, recent studies indicated local consolidative therapy were able to prolong progression-free survival and overall survival (OS) in stage IV NSCLC patients who received first-line systemic therapy $(5,6)$. Nevertheless, the role of primary lesion surgery in patients with occult Mla lung cancer receiving targeted therapy remains unclear.

To address this, we aimed to assess OS after primary tumor resection versus no resection in lung adenocarcinoma patients with occult pleural dissemination treated with EGFR-TKIs.

\section{METHODS}

\section{Patients}

Selected patients with occult M1a lung adenocarcinoma hospitalized in the Department of Thoracic Surgery, Fudan Shanghai Cancer Center (FUSCC) from May 2008 to December 2017 were reviewed retrospectively. The inclusion criteria were (1) pathologically confirmed primary lung adenocarcinoma, (2) occult pleural dissemination and pathologically confirmed M1a intraoperatively by frozen section examinations, and (3) receiving targeted therapy toward EGFR mutation. Age, gender, smoking history, body mass index (BMI), receiving primary tumor resection or not, mutation status, EGFR-TKIs therapy, and survival data were collected. Primary tumor resection was defined as the surgical removal of primary lung cancer lesion, which was usually the largest or first appeared radiologically. The study was approved by the Institutional Review Board of Fudan University Shanghai Cancer Center (IRB\#090977-1), and the protocol number of this study was IRB2008223-9.

\section{Mutational Analyses}

The mutational analyses were conducted by the central laboratory of pathology in FUSCC using the resected primary tumor specimens. Genomic DNA was extracted for further amplification refractory mutation system.

\section{Statistical Analyses}

Overall survival was calculated from the date of the diagnosis to the date of death or last follow-up, and death from any cause was considered as an event. Chi-square tests were used to evaluate the difference of categorized variables between patients receiving and not receiving primary tumor resection. The Kaplan-Meier method was used to analyze OS, and log-rank tests were used to compare differences between groups. Data were analyzed by SPSS (version 25.0; IBM Corp, Armonk, NY). All tests were twotailed, and statistical significance was set at $\mathrm{P}<0.05$.

\section{RESULTS}

\section{Patient Characteristics}

A total of 34 patients were enrolled in the study (Table 1). There are $22(64.7 \%)$ females and 12 (35.3) males. A majority of patients $(29 / 34,85.3 \%)$ were never-smokers. Most of patients had cT1 and cT2 disease. $17(50.0 \%)$ patients had cN0 disease, 5 (14.7\%) had $\mathrm{cN} 1$, and 12 (35.3\%) had $\mathrm{cN} 2$ disease. Among these patients, 20 (58.8\%) patients underwent primary tumor resection, while the rest (14/34, 41.2\%) not. Concerning firstly-used EGFR-TKIs, the most common agent was gefitinib $(26 / 34,67.2 \%)$, followed by erlotinib $(6 / 34$, $17.6 \%)$, icotinib $(1 / 34,2.9 \%)$, and osimertinib $(1 / 34,2.9 \%)$. Five $(5 / 34,14.7 \%)$ patients received osimertinib as the second therapy after developing drug resistance to gefitinib and erlotinib. Besides targeted therapy, 23 (67.6\%) patients received platinum doublet chemotherapy, and $6(17.6 \%)$ patients received radiotherapy.

Comparisons were made between patients who received primary tumor resection and those who did not (Table 2). There were no distributional differences between two groups in age $(P=0.477)$, gender $(P=0.066)$, smoking history $(P=1.000)$, body mass index (BMI, $P=1.000), \mathrm{cT}(P=0.207), \mathrm{cN}(P=0.512)$, firstly-used EGFR-TKIs $(P=1.000)$, secondly-used osimertinib $(P=0.627)$, platinum doublet chemotherapy $(P=1.000)$, and radiotherapy $(P=1.000)$. Previous studies have revealed the prognostic value of clinical $\mathrm{T}$ and $\mathrm{N}$ descriptors in patients with operable lung cancer $(7,8)$, so we also investigated it in patients with intraoperatively-confirmed M1a disease. The results demonstrated that there was no difference in survival between patients with different $\mathrm{cT}(P=0.96)$ and $\mathrm{cN}(P=0.87)$ descriptors (Supplementary Figure 1). 
TABLE 1 | Clinicopathologic characteristics of patients with M1a lung adenocarcinoma.

\begin{tabular}{|c|c|c|}
\hline \multirow[t]{2}{*}{ Variables } & \multicolumn{2}{|c|}{ Enrolled patients $(\mathrm{N}=34)$} \\
\hline & $\mathbf{N}$ & $\%$ \\
\hline \multicolumn{3}{|l|}{ Age (years) } \\
\hline$\leq 60$ & 21 & 61.8 \\
\hline$>60$ & 13 & 38.2 \\
\hline \multicolumn{3}{|l|}{ Gender } \\
\hline Male & 12 & 35.3 \\
\hline Female & 22 & 64.7 \\
\hline \multicolumn{3}{|l|}{ Smoking history } \\
\hline Ever & 5 & 14.7 \\
\hline Never & 29 & 85.3 \\
\hline \multicolumn{3}{|l|}{ BMI } \\
\hline$\leq \mathbf{2 4}$ & 23 & 67.6 \\
\hline$>24$ & 11 & 32.4 \\
\hline \multicolumn{3}{|l|}{ cT } \\
\hline cT1 & 14 & 41.2 \\
\hline сT2 & 18 & 52.9 \\
\hline сT3 & 1 & 2.9 \\
\hline cT4 & 1 & 2.9 \\
\hline \multicolumn{3}{|l|}{$\mathrm{cN}$} \\
\hline cNO & 17 & 50.0 \\
\hline cN1 & 5 & 14.7 \\
\hline cN2 & 12 & 35.3 \\
\hline \multicolumn{3}{|c|}{ Primary tumor resection } \\
\hline Yes & 20 & 58.8 \\
\hline No & 14 & 41.2 \\
\hline \multicolumn{3}{|c|}{ Firstly-used EGFR-TKIs } \\
\hline Gefitinib & 26 & 76.5 \\
\hline Erlotinib & 6 & 17.6 \\
\hline Icotinib & 1 & 2.9 \\
\hline Osimertinib & 1 & 2.9 \\
\hline \multicolumn{3}{|c|}{ Secondly-used osimertinib } \\
\hline Yes & 5 & 14.7 \\
\hline No & 29 & 85.3 \\
\hline \multicolumn{3}{|c|}{ Platinum doublet chemotherapy } \\
\hline Yes & 23 & 67.6 \\
\hline No & 11 & 32.4 \\
\hline \multicolumn{3}{|l|}{ Radiotherapy } \\
\hline Yes & 6 & 17.6 \\
\hline No & 28 & 82.4 \\
\hline
\end{tabular}

BMI, Body Mass Index.

16 patients died during the follow-up period. With the median follow-up time of 65.0 months, the median OS was 60.0 months (95\% confidential interval [CI], 33.4-88.6). The 2 -year and 5-year OS was $85.3 \%$ and $45.5 \%$ respectively (Figure 1).

\section{The Association Between Primary Tumor Resection and Improved Survival}

To investigate the prognostic role of primary tumor resection, Kaplan-Meier curves were used. The patients undergoing primary tumor resection had a prolonged OS compared with those not $(\log$-rank $P=0.042$; Figure 2). The median $O S$ for patients receiving primary tumor resection and those not was 83.0 months (95\% CI: 41.2-124.8) and 43.7 months (95\%CI: 30.6-56.7). The 2-year and 5-year OS for patients receiving primary tumor resection was $90.0 \%$ and $60.1 \%$, while the 2 year and 5-year OS for those not was $78.6 \%$ and $26.8 \%$.

\section{DISCUSSION}

Currently, there was no consensus on whether to perform surgical resection to lung cancer patients with occult pleural disseminations. Chemotherapy or targeted therapy was recommended as initial treatments for stage IV NSCLC, and radiotherapy could also be considered if necessary, without the recommendation of surgical resection. Gomez and his colleges $(5,6)$ reported local consolidative therapy, including surgery and/or radiotherapy, could prolong progression-free survival and overall survival significantly in stage IV lung cancer after receiving first-line systemic therapy. However, only patients with stable disease and partial response to chemotherapy were enrolled, and most of the patients received platinum doublet chemotherapy as the first-line treatment. Besides, there were only six patients $(6 / 25,24 \%)$ receiving surgery of metastatic and/ or primary sites in their study. Therefore, the actual effect of surgery in patients with stage IV lung cancer remains unclear, especially for patients receiving targeted therapy. In our study, we investigated lung adenocarcinoma patients with occult pleural disseminations receiving EGFR-TKIs and found out upfront primary tumor resection was associated with improved survival. To the best of our knowledge, it is the first study to reveal the association between primary surgical resection and improved survival in lung adenocarcinoma patients with occult pleural metastases in the era of targeted therapy. The study provided novel evidence for the treatments of occult M1a lung adenocarcinoma.

In clinical practice, pleural disseminations might be occult for some patients and was discovered intraoperatively. There is no consensus on whether we should perform primary tumor resection in that case. Since surgical resection can result in a 5year survival rate of $30 \%$ to $50 \%$ in patients with metastatic NSCLC (9), and the role of thoracic surgery in the management of metastatic NSCLC attracts our attention. Theoretically, surgical resection of primary lesions could reduce tumor burden, which was considered to be associated with targeted drug resistance and prognosis of patients. In our study, primary tumor resection could significantly prolong the survival of patients receiving EGFR-TKIs $(P=0.042)$. The 5 -year OS of patients undergoing surgical resection and those not was $60.1 \%$ and $26.8 \%$. These results supported the conclusion that surgical resection could improve survival in lung adenocarcinoma patients with occult pleural disseminations receiving EGFR-TKIs.

With the improved disease response and control rate with targeted therapy, patients with advanced-stage lung cancer live longer. According to previous real-world studies (10), the median OS for EGFR-mutant metastatic lung adenocarcinoma patients treated with EGFR-TKIs was 30.9 months. Especially for Asian patients, our previous study more than half of patients with lung adenocarcinoma harbored EGFR kinase domain mutations (11). The targeted agent is an ideal treatment for them, but drug resistance will occur sooner or later. Our study might provide a potential way to slow down the drug resistance to EGFR-TKIs. Further randomized clinical trials are urged on the possible combined use of surgery and targeted therapy. 
TABLE 2 | Clinicopathologic characteristics of patients receiving and not receiving primary lesion resection.

\begin{tabular}{|c|c|c|c|c|c|}
\hline \multirow[t]{2}{*}{ Variables } & \multicolumn{2}{|c|}{ Patients receiving primary tumor resection $(N=20)$} & \multicolumn{2}{|c|}{ Patients not receiving resection $(\mathrm{N}=14)$} & \multirow[t]{2}{*}{$P$} \\
\hline & $\mathbf{N}$ & $\%$ & $\mathbf{N}$ & $\%$ & \\
\hline Age (years) & & & & & 0.477 \\
\hline$\leq 60$ & 11 & 55.0 & 10 & 71.4 & \\
\hline$>60$ & 9 & 45.0 & 4 & 28.6 & \\
\hline Gender & & & & & 0.066 \\
\hline Male & 10 & 50.0 & 12 & 85.7 & \\
\hline Female & 10 & 50.0 & 2 & 14.3 & \\
\hline Smoking history & & & & & 1.000 \\
\hline Ever & 3 & 15.0 & 2 & 14.3 & \\
\hline Never & 17 & 85.0 & 12 & 85.7 & \\
\hline BMI & & & & & 1.000 \\
\hline$\leq \mathbf{2 4}$ & 14 & 70.0 & 9 & 64.3 & \\
\hline$>24$ & 6 & 30.0 & 5 & 35.7 & \\
\hline cT & & & & & 0.207 \\
\hline cT1 & 6 & 30.0 & 8 & 57.1 & \\
\hline сT2 & 13 & 65.0 & 5 & 35.7 & \\
\hline cT3/4 & 1 & 5.0 & 1 & 7.1 & \\
\hline $\mathrm{cN}$ & & & & & 0.512 \\
\hline cNO & 10 & 50.0 & 7 & 50.0 & \\
\hline $\mathrm{cN} 1$ & 4 & 20.0 & 1 & 7.1 & \\
\hline cN2 & 6 & 30.0 & 6 & 42.9 & \\
\hline Firstly-used EGFR-TKIs & & & & & 1.000 \\
\hline Gefitinib & 15 & 75.0 & 11 & 78.6 & \\
\hline Erlotinib & 3 & 15.0 & 3 & 21.4 & \\
\hline Icotinib & 1 & 5.0 & 0 & 0 & \\
\hline Osimertinib & 1 & 5.0 & 0 & 0 & \\
\hline Secondly-used osimertinib & & & & & 0.627 \\
\hline Yes & 2 & 10.0 & 3 & 21.4 & \\
\hline No & 18 & 90.0 & 11 & 78.6 & \\
\hline Platinum doublet hemotherapy & & & & & 1.000 \\
\hline Yes & 14 & 70.0 & 9 & 64.3 & \\
\hline No & 6 & 30.0 & 5 & 35.7 & \\
\hline Radiotherapy & & & & & 1.000 \\
\hline Yes & 3 & 15.0 & 3 & 21.4 & \\
\hline No & 17 & 85.0 & 11 & 78.6 & \\
\hline
\end{tabular}

BMI, Body Mass Index.

The survival benefit from surgical resection of primary tumors might be explained by the following potential mechanisms. Intratumor heterogeneity results in different subclones of tumor cells, some of which are resistant to targeted therapy or chemotherapy (12). After resection of the primary lesion, generally the largest of all lesions, the drugresistant subclones could also be removed (13). Thus, in this scenario, patients may have a better drug response and longer survival. Another possible mechanism is that primary tumors seed circulating tumor cells via the bloodstream, resulting in micro-metastasis in distant sites (14). In that case, resection of primary tumors could slow the growth speed of micrometastasis. Therefore, upfront surgical resection helped to maximize drug response of targeted therapy, suggesting a combination of surgical resection and targeted therapy might be an effective option.

In our study, surgical resection was performed before targeted therapy. Upfront surgery followed by targeted therapy could provide advantages in some ways. In our previous study, upfront surgery followed by adjuvant therapy may also provide favorable survival outcomes for selected patients with lung cancer (15).
The resection of primary lesions spared patients from biopsies to confirm pathology and mutational status. Additionally, pathologic and mutational analyses based on surgically resected specimens were generally more precise than biopsy specimens. Therefore, upfront surgery followed by targeted therapy was feasible in clinical practice.

There are several limitations of this study. First, the number of patients seemed small. We only enrolled patients receiving EGFR-TKIs with intraoperatively-confirmed occult M1a lung adenocarcinoma, who have been hospitalized in the department of thoracic surgery. The multivariable analyses were inappropriate due to limited sample size. Nevertheless, the baselines of two groups were comparable, resulting in no confounding factors during the direct survival comparison by Kaplan-Meier method. Second, it is a retrospective study from a single institution, and selection bias was inevitable. Our results need to be validated in future multi-centered randomized controlled clinical trials. Third, progression-free survival was not calculated in the study, because some patients received surveillance in other institutions. Nevertheless, we believe that OS is a more important outcome and the study based on OS is 


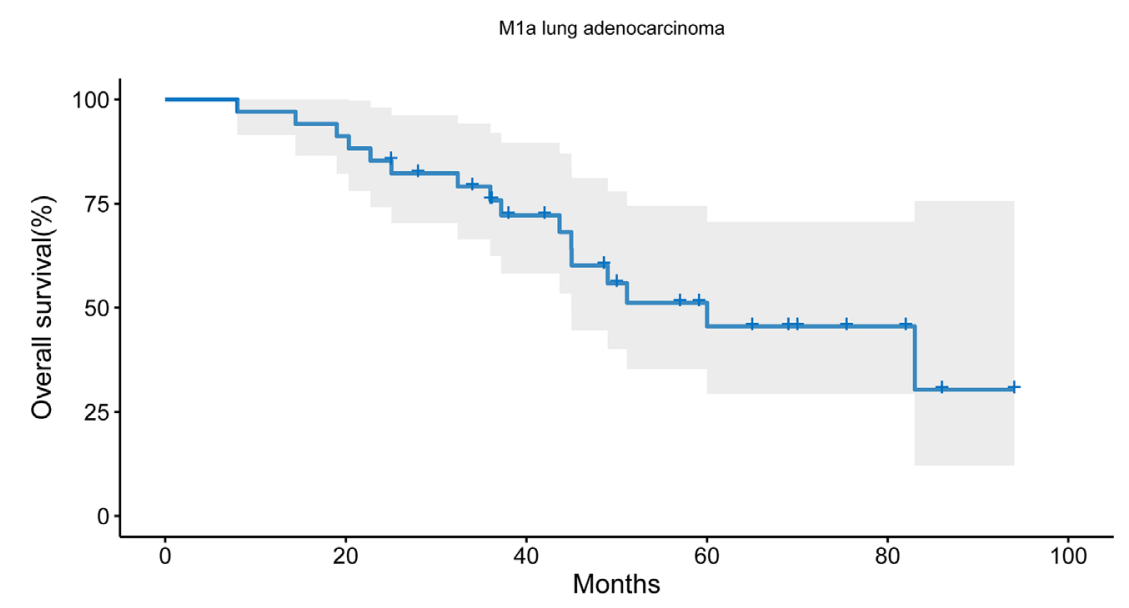

Number at risk

\begin{tabular}{|c|c|c|c|c|c|}
\hline 34 & 31 & 19 & 9 & 4 & 0 \\
\hline 0 & 20 & 40 & 60 & 80 & 100 \\
\hline
\end{tabular}

FIGURE 1 | Overall survival of M1a lung adenocarcinoma patients receiving targeted therapy.

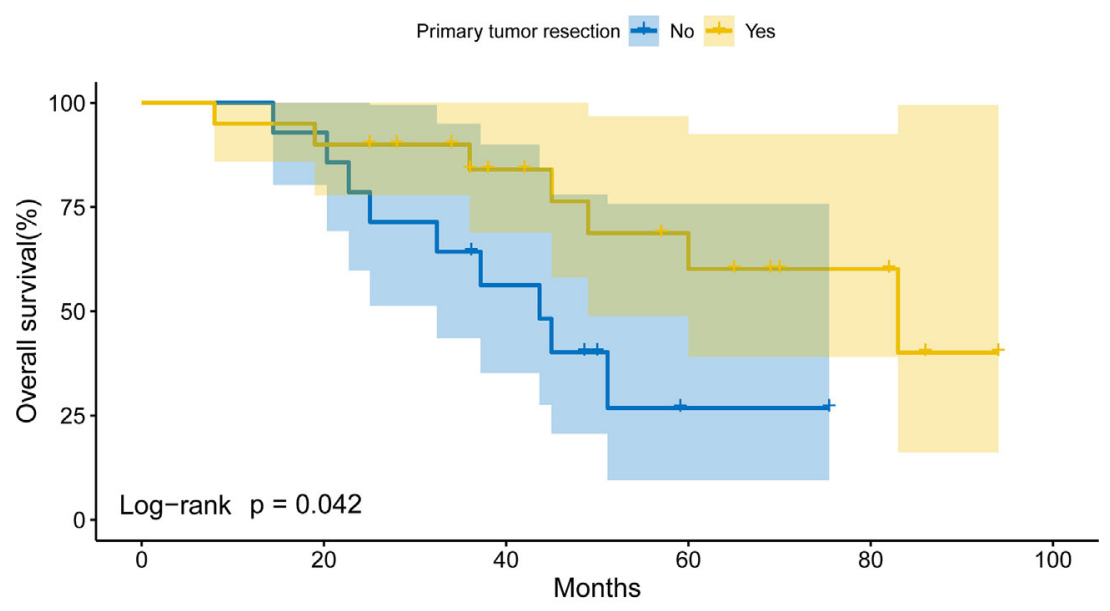

Number at risk

\begin{tabular}{|c|c|c|c|c|c|c|}
\hline No & 14 & 13 & 7 & 1 & 0 & 0 \\
\hline Yes. & 20 & 18 & 12 & 8 & 4 & 0 \\
\hline & 0 & 20 & 40 & 60 & 80 & 100 \\
\hline
\end{tabular}

FIGURE 2 | Overall survival of EGFR-TKIs-treated patients with intraoperatively-confirmed M1a lung adenocarcinoma receiving or not receiving primary tumor resection.

more meaningful for patients. Fourth, the main agent of EGFRTKIs in this study was gefitinib due to the use of histological data before the adoption of first-line osimertinib. However, the study provided a promising treatment for lung adenocarcinoma with occult pleural disseminations.
In summary, primary surgical resection improves survival of lung adenocarcinoma patients with intraoperatively-confirmed occult pleural metastases followed by EGFR-TKIs. Primary tumor resection might be a promising method for the treatment of patients with occult M1a lung adenocarcinoma receiving target therapy. 


\section{DATA AVAILABILITY STATEMENT}

The original contributions presented in the study are included in the article/Supplementary Material. Further inquiries can be directed to the corresponding authors.

\section{ETHICS STATEMENT}

The studies involving human participants were reviewed and approved by Institutional Review Board of Fudan University Shanghai Cancer Center (IRB\#090977-1). Written informed consent for participation was not required for this study in accordance with the national legislation and the institutional requirements.

\section{AUTHOR CONTRIBUTIONS}

Study concepts: FF, ZW, YaZ, and HC. Study design: FF, ZW, YaZ, and HC. Literature research: FF, ZW, ZG, YuZ, YL, YaZ, and HC. Data acquisition: FF, ZW, ZG, YuZ, and YL. Data analysis/ interpretation: FF, ZW, ZG, YuZ, and YL. Statistical analysis: FF, ZW, ZG, YuZ, and YL. Manuscript preparation: FF, ZW, YaZ, and

\section{REFERENCES}

1. Bray F, Ferlay J, Soerjomataram I, Siegel RL, Torre LA, Jemal A. Global cancer statistics 2018: GLOBOCAN estimates of incidence and mortality worldwide for 36 cancers in 185 countries. CA Cancer J Clin (2018) 68:394-424. doi: 10.3322/caac.21492

2. Chen VW, Ruiz BA, Hsieh MC, Wu XC, Ries LA, Lewis DR. Analysis of stage and clinical/prognostic factors for lung cancer from SEER registries: AJCC staging and collaborative stage data collection system. Cancer (2014) 120:3781-92. doi: 10.1002/cncr.29045

3. Goldstraw P, Chansky K, Crowley J, Rami-Porta R, Asamura H, Eberhardt WE, et al. The IASLC Lung Cancer Staging Project: Proposals for Revision of the TNM Stage Groupings in the Forthcoming (Eighth) Edition of the TNM Classification for Lung Cancer. J Thorac Oncol (2016) 11:39-51. doi: 10.1016/j.jtho.2015.09.009

4. Al-Halabi H, Sayegh K, Digamurthy SR, Niemierko A, Piotrowska Z, Willers $\mathrm{H}$, et al. Pattern of Failure Analysis in Metastatic EGFR-Mutant Lung Cancer Treated with Tyrosine Kinase Inhibitors to Identify Candidates for Consolidation Stereotactic Body Radiation Therapy. J Thorac Oncol (2015) 10:1601-7. doi: 10.1097/JTO.0000000000000648

5. Gomez DR, Blumenschein GRJr, Lee JJ, Hernandez M, Ye R, Camidge DR, et al. Local consolidative therapy versus maintenance therapy or observation for patients with oligometastatic non-small-cell lung cancer without progression after first-line systemic therapy: a multicentre, randomised, controlled, phase 2 study. Lancet Oncol (2016) 17:1672-82. doi: 10.1016/S1470-2045(16)30532-0

6. Gomez DR, Tang C, Zhang J, Blumenschein GRJr, Hernandez M, Lee JJ, et al. Local Consolidative Therapy Vs. Maintenance Therapy or Observation for Patients With Oligometastatic Non-Small-Cell Lung Cancer: Long-Term Results of a Multi-Institutional, Phase II, Randomized Study. J Clin Oncol (2019) 37:1558-65. doi: 10.1200/JCO.19.00201

7. Tsutani Y, Miyata Y, Nakayama H, Okumura S, Adachi S, Yoshimura M, et al. Solid tumor size on high-resolution computed tomography and maximum standardized uptake on positron emission tomography for new clinical $\mathrm{T}$ descriptors with T1 lung adenocarcinoma. Ann Oncol (2013) 24:2376-81. doi: 10.1093/annonc/mdt230

8. Suzuki K, Nagai K, Yoshida J, Nishimura M, Takahashi K, Nishiwaki Y. The prognosis of surgically resected N2 non-small cell lung cancer: the importance
HC. Manuscript editing: FF, ZW, YaZ, and HC. Manuscript final version approval: FF, ZW, ZG, YuZ, YL, YaZ, and HC. All authors contributed to the article and approved the submitted version.

\section{FUNDING}

This work was supported by the National Natural Science Foundation of China (81930073 and 81772466), Shanghai Science and Technology Innovation Action Project (20JC1417200), Shanghai Municipal Science and Technology Major Project (2017SHZDZX01, VBH1323001/026), Shanghai Municipal Key Clinical Specialty Project (SHSLCZDZK02104), and Pilot Project of Fudan University (IDF159045).

\section{SUPPLEMENTARY MATERIAL}

The Supplementary Material for this article can be found online at: https://www.frontiersin.org/articles/10.3389/fonc.2021.622723/ full\#supplementary-material

Supplementary Figure 1 | Overall survival of patients with intraoperativelyconfirmed M1a lung adenocarcinoma stratified by cT (A) and cN (B).

of clinical N status. J Thorac Cardiovasc Surg (1999) 118:145-53. doi: 10.1016/ S0022-5223(99)70153-4

9. David EA, Clark JM, Cooke DT, Melnikow J, Kelly K, Canter RJ. The Role of Thoracic Surgery in the Therapeutic Management of Metastatic Non-Small Cell Lung Cancer. J Thorac Oncol (2017) 12:1636-45. doi: 10.1016/ j.jtho.2017.08.008

10. Lin JJ, Cardarella S, Lydon CA, Dahlberg SE, Jackman DM, Janne PA, et al. Five-Year Survival in EGFR-Mutant Metastatic Lung Adenocarcinoma Treated with EGFR-TKIs. J Thorac Oncol (2016) 11:556-65. doi: 10.1016/ j.jtho.2015.12.103

11. Wang R, Zhang Y, Pan Y, Li Y, Hu H, Cai D, et al. Comprehensive investigation of oncogenic driver mutations in Chinese non-small cell lung cancer patients. Oncotarget (2015) 6:34300-8. doi: 10.18632/oncotarget.5549

12. Cheng X, Chen H. Tumor heterogeneity and resistance to EGFR-targeted therapy in advanced nonsmall cell lung cancer: challenges and perspectives. Onco Targets Ther (2014) 7:1689-704. doi: 10.2147/OTT.S66502

13. Hittelman WN. Clones and subclones in the lung cancer field. J Natl Cancer Inst (1999) 91:1796-9. doi: 10.1093/jnci/91.21.1796

14. Mascaux C, Angelova M, Vasaturo A, Beane J, Hijazi K, Anthoine G, et al. Immune evasion before tumour invasion in early lung squamous carcinogenesis. Nature (2019) 571:570-5. doi: 10.1038/s41586-019-1330-0

15. Zheng D, Ye T, Hu H, Zhang Y, Sun Y, Xiang J, et al. Upfront surgery as firstline therapy in selected patients with stage IIIA non-small cell lung cancer. J Thorac Cardiovasc Surg (2018) 155:1814-22. doi: 10.1016/j.jtcvs.2017.10.075

Conflict of Interest: The authors declare that the research was conducted in the absence of any commercial or financial relationships that could be construed as a potential conflict of interest.

Copyright (c) $2021 \mathrm{Fu}$, Wen, Gao, Zhao, Li, Zhang and Chen. This is an open-access article distributed under the terms of the Creative Commons Attribution License (CC BY). The use, distribution or reproduction in other forums is permitted, provided the original author(s) and the copyright owner(s) are credited and that the original publication in this journal is cited, in accordance with accepted academic practice. No use, distribution or reproduction is permitted which does not comply with these terms. 